Original Research Paper

\title{
On a New Equation for the Design and Development of Space Launch Vehicles
}

\author{
Zaheen Abdur Rahman
}

Space Engineering, Aerospace and Astronautics, University Campus, Dhaka, Bangladesh

\section{Article history}

Received: 16-01-2018

Revised: $10-02-2018$

Accepted: 22-02-2018

E-mail: zaheenarahman@gmail.com

\begin{abstract}
It is a general consensus amongst the global scientific community that space is the final frontier for human exploration. It has also been reasoned by them that in order to survive in this hostile universe, humanity has to become a multi-planetary species. For that reason, according to calculations made after mapping the human genome, over 10,000 people are needed to start off an independent space colony, taking into account the inhospitality of space and human beings' relatively slow speed of evolution in such conditions. As of date, there have been very few equations regarding this issue, such as the Tsiolkovsky Rocket Equation, and the author regards his equation to be the pinnacle of space launch vehicle design. Taking into consideration the costs of carrying so many people and their supplies, it would be enormously helpful if a single equation could be used to develop the whole rocket, as it would not only reduce development and testing costs, but launch costs as well, and that is exactly what this article intends to provide.
\end{abstract}

Keywords: Drag, Density, Energy, Equation, Fuel, Mass, Velocity

\section{Introduction}

Currently, the design and construction of rockets typically involves thousands of hours of research, modelling and finally, testing. Along with that, considering our goal is the creation of an $\mathrm{L}_{5}$ colony (NASA, 1977), this involves a massive workforce and is thus an extremely timeconsuming and arduous task, implying that a huge sum of money is required. Therefore, considering the highly fruitful implications of spending less money and time on one project of "the exploration of outer space", such as more projects for the same amount of money and an increase in the speed of space exploration, I believe it is highly necessary to find a complete equation for the design and development of Space Launch Vehicles.

In order to obtain the equation, a number of methods were used. First and foremost was numerical analysis, as shall be explained in the next section. Then, a few extra equations were created, using the principles of density, the volumes and surface areas of different shapes such as cones and right circular cylinders and air resistance. Lastly, all of them were combined together in an accurate manner to form one, single, elegant equation.

\section{The Base Equations}

The equation is mainly based upon that for kinetic energy, namely:

$$
E_{K}=\frac{m V^{2}}{2}
$$

\section{Where:}

$$
\begin{aligned}
& E_{K}=\text { The kinetic energy } \\
& m=\text { The mass and } \\
& \mathrm{V}^{2}=\text { The average velocity squared in }\left(\mathrm{m} \mathrm{s}^{-1}\right)^{2}
\end{aligned}
$$

The first addition to this is the equation of the atmospheric effects of drag, which is divided by one second (since the value is for one second, we have to obtain drag $(\mathrm{N})$ in $\mathrm{N} / \mathrm{s}$ ), then multiplied by the time taken by the rocket to reach the altitude of engine burnout (found by dividing altitude by average velocity) and finally divided by the acceleration ${ }^{1}$, $19.6133 \mathrm{~m} \mathrm{~s}^{-2}$. Thus we find drag in kilograms (Johnson and Cutnell, 1995). This, therefore, can be added to mass, as it, too, like mass, is a form of resistance. Accordingly, the completed drag equation is (original drag equation/1 second * time till engine burnout)/(acceleration) (as modified from Hitchens, 2015) or:

$$
D=\frac{C_{D} * 0.5 * \rho * V^{2} * A * S_{r}}{19.6133}
$$


Where:

$D=$ The final drag

$C_{D}=$ The coefficient of drag

$\rho=$ Is the density in $\mathrm{kg} \mathrm{m}^{-3}$

$A=$ The total outer surface area of the rocket in $\mathrm{m}^{2}$ and

$s_{r}=$ The time till engine burnout (with no unit, since the $s$ of $1 \mathrm{sec}$ and the $s$ of $s_{r}$ cancel each other out)

So, currently, after rearrangement, our equation stands at:

$$
\frac{(2 * E K)}{V^{2}}=m+\frac{C_{D} * 0.5 * \rho * V^{2} * A * s_{r}}{19.6133}
$$

Hereafter we need to go into the finer details of the equation. First and foremost is the measurement of $E_{K}$. To do this, the heating value of the fuel is measured in $J$ $\mathrm{kg}^{-1}$. Then, it is multiplied by the time till engine burnout $\left(s_{r}\right)$, as our goal is to find the kinetic energy in total. Thereafter we get $J \mathrm{~kg}^{-1} * s_{r}$. Then, we have to multiply this value with the kilograms of fuel being burnt in one second (in $\mathrm{kg} \mathrm{s}^{-1}$ ), finally giving us the value in Joules. So, finally, we get: $J \mathrm{~kg}^{-1} * s_{r} * \mathrm{~kg} \mathrm{~s}^{-1}$. As the ' $\mathrm{kg}^{\prime}$ and 's' units cancel each other out, we get the total energy in Joules. Therefore, after the addition of engine efficiency $E$, our equation stands at:

$$
\frac{E * 2 * J \mathrm{~kg}^{-1} * s_{r} * \mathrm{~kg} \mathrm{~s}^{-1}}{V^{2}}=m+\frac{C_{D} * 0.5 * \rho * V^{2} * A * s_{r}}{19.6133}
$$

Where:

$E \quad=$ The efficiency of the engines

$J \mathrm{~kg}^{-1}=$ Signifies the heating value

$\mathrm{kg} \mathrm{s}^{-1}=$ The kilograms of fuel burnt in one second

In this paragraph, we shall concentrate on average velocity and the time till the engine burnout. The equation for average velocity is relatively easy. It is, effectively, a solution of the equation for the maximum height attained by a rocket that has shut down its engines (Silver, 2009):

$$
h=h_{0}+\frac{(v \sin \theta)^{2}}{2 g}
$$

Where:

$h=$ Final height

$h_{0}=$ Height at burn-out

$v=$ Speed at burnout

$\theta=$ Angle at launch and

$g=$ Acceleration due to gravity

Fortunately, the limit for ' $\mathrm{g}$ ' forces on human space vehicles has been set at 3 g's (NASA, 2002), while the angle at launch $(\theta)$ is always 90 degrees, meaning $\sin (\theta)$ is equal to 1 . Therefore we use the series $\frac{n(n+1)}{2}$ to find the height of the rocket at burnout, with the result being multiplied by $2 g$ (as Earth's pull is $1 \mathrm{~g}$, leaving $2 \mathrm{~g}$ 's). The speed, of course, is $2 g n$, as $n$ is the time elapsed since launch in seconds. As for the final height, it is, obviously, the orbital altitude (in metres). So, we get the equation:

$h=g n(n+1)+2 g n^{2}$

As one may already have identified, $n$ is the time taken till the engine burnout and is therefore equal to $s_{r}$.

Now we come to our penultimate and most important step: Mass. In order to find mass, we have to first take the known weights, that is, the parts with no involvement with fuel whatsoever, the mass of the astronauts, their gear, communications equipment, satellites, the top and bottom areas of the fuel tanks and the weights of the engines, times the density of the material used to make the spacecraft. While the others may be easily found, the fuel tanks' volumes have to be calculated by an equation. That equation, if the fuel tanks are on top of each other and separated by a divider $^{2}$, as in Saturn V's first stage, with half-spheres at the top and bottom and cylinder in between, is:

$\frac{m}{D}=\frac{4 \pi R^{3}}{3}+\pi R^{2} h$

\section{Where:}

$m=$ The total mass of the fuel

$D=$ The average density of the fuel

$R=$ The inner radius and

$h=$ The height of the cylinder ${ }^{3}$

If the simplification is done according to the endnote, the equation becomes

$\frac{m}{D}=\frac{4 \pi R^{3}}{3}+\frac{(m-M)}{D}$

Where:

$M=$ The mass of fuel in the sphere ${ }^{4}$

If the shape of the fuel tank is just a cylinder with a divider, the equation simply becomes:

$$
\frac{m}{D}=\pi R^{2} h
$$

with the divider being placed at $\frac{A_{r} m}{\pi R^{2} D M_{r}}$ and the variables being the same as those in Equation 7 and 8 and endnotes 2 and 4 above. 
Having found the volumes, the masses of these cylinders and spheres (if any) are easily found by multiplying the volume of the material covering them with the density of that material, in other words, using the equation:

$$
\frac{4 d \pi\left(r^{3}-R^{3}\right)}{3}=M_{1}
$$

Where:

$r \quad=$ The outer radius

$d=$ The density of the material used to make the cylinder and

$M_{1}=$ The final mass of the materials used to make the sphere

As one might have guessed, $M_{2}$ is for the final mass of the materials used to make the cylinder, with the equation for a sphere-attached cylinder and divider being:

$$
\frac{\left(r^{2}-R^{2}\right)(m-M)(d)}{D R^{2}}+V_{1}=M_{2}
$$

and that for a non-sphere-attached cylinder and divider being:

$$
\frac{\left(r^{2}-R^{2}\right)(m)(d)}{D R^{2}}+V_{1}=M_{2}
$$

Where, in both cases:

$V_{1}=$ Mass of the divider ${ }^{5}$ and

$M_{2}=$ The final mass of the materials used to make the cylinder

Of course:

$$
M_{1}+M_{2}+0.5 m=M_{3}
$$

Where ${ }^{6}$ :

$M_{3}=$ The total mass of the fuel tank and the fuel in it, that is, the total unknown mass

Therefore, we have completed the part of unknown mass and must now move on to a short paragraph on the steps to finding the known mass.

Firstly, the shape of the payload bay or astronaut quarters or both is determined.

Then, the thickness of the wall holding it/them is decided.

After that, height is found out, according to prior measurements of the average requirement of space for an astronaut and his supplies over a finite amount of time, or the density of payloads in the bay, or both.
Finally, the volume of the total structure is uncovered and multiplied by the density of its construction material to obtain its mass and that is added to the masses of the astronauts, their supplies, the air in that area and the engines. The variable for the total known mass is $F_{M}$.

Having ended our inquiry of mass at this point, we can now move on to our final requirement: drag. The equation of drag is, as has already been mentioned earlier, Equation 2. Now, considering that in order to find the average coefficient of drag, we have to multiply individual fractions of the nature of shape/total area by its coefficient of drag. We have a huge advantage in this regard: the reference area and the denominator of the coefficient of drag cross each other out, leaving us with the numerator of $C_{D}$, to be multiplied with $0.5, V^{2}, \rho$ and $s_{r}$ and the result to be divided by 19.6133 . As a recap, $V^{2}$ is found by $g(n+1)\left(=g\left(s_{r}+1\right)\right)^{7}$ and $s_{r}$ is found as $n$ using Equation 6.

Lastly, $\rho$, the density of air, is found by simply dividing the density of air at sea level by two ${ }^{8}$.

\section{The Proposed Equation}

Therefore our final equation is:

$$
\begin{aligned}
& \frac{E^{*} 2 * J \mathrm{~kg}^{-1} * s_{r} * k g s^{-1}}{V^{2}}=\frac{4 \pi r_{1}^{3} R_{1}^{3} d_{1}}{3}+\frac{(m-M)\left(r_{2}^{2}-R_{2}^{2}\right) d_{2}}{D R^{2}} \\
& +\pi d_{3} r_{1}^{2}+0.5 m+F_{M}+\frac{C_{D} * 0.5 * \rho^{*} V^{2} * A^{*} s_{r}}{19.6133} \\
& \rightarrow \frac{E * 2 * J \mathrm{~kg}^{-1} * m}{V^{2}}=M_{1}+M_{2}+0.5 m+F_{M} \\
& +\frac{C_{D n} * 0.5 * \rho * V^{2} * s_{r}}{19.6133} \\
& \rightarrow \frac{E * 2 * J \mathrm{~kg}^{-1} * m}{V^{2}}=M_{3}+F_{M}+D_{M} \\
& \rightarrow E_{E O}=T_{E M} V^{2}
\end{aligned}
$$

Where:

$E \quad=$ The engine efficiency

$J \mathrm{~kg}^{-1}=$ The heating value of the fuel

$s_{r} \quad=$ The time taken till engine burnout

$\mathrm{kg} \mathrm{s}^{-1}=$ The amount of fuel used per second

$V=$ The average velocity

$r_{1}=$ The outer radius of the sphere

$R_{1} \quad=$ The inner radius of the sphere

$d_{1}=$ The density of the material used to make the sphere

$m \quad=$ The mass of fuel in the rocket

$\mathrm{M}=$ The mass of fuel in the sphere

$r_{2}=$ The outer radius of the cylinder

$h \quad=$ The height of the divider 
$d_{2}=$ The density of the material used to make the cylinder

$D \quad=$ The density of the fuel

$R_{2} \quad=$ The inner radius of the cylinder

$d_{3}=$ The density of the material used to make the divider

$C_{D} \quad=$ The coefficient of drag

$\rho \quad=$ The density of the air

$\mathrm{A}=$ The total area of the rocket

$M_{1}=$ The mass of the sphere

$M_{2}=$ The mass of the cylinder and divider

$F_{m} \quad=$ The known mass

$C_{D n}=$ The numerator of the average coefficient of drag

$M_{3}=$ The total unknown mass in the rocket

$D_{M}=$ The mass of the drag on the rocket

$E_{E O}=$ The effective engine output by average velocity and

$T_{E M}=$ The total effective mass

\section{Discussion}

The equation has to be worked backwards, that is, first it has to be used for stage 3, then stage 2 and then stage 1 , after which the three m's can be added together for total fuel. This is because this equation cannot handle more than one unknown variable of the same kind at once. Also, any additional fuel for necessary deceleration, acceleration, or boosters is to be added to the fixed mass and can be found by the concept of ratios:

$\frac{X}{P}=\frac{Y}{100}$

Where:

$X=$ The mass of the fuel and boosters required

$P \quad=$ The payload

$Y=$ The mass of the fuel and boosters per 100 kilograms of payload and

$100=$ taken as the base mass, as $\frac{Y}{100}$ can also be expressed as $Y \%$ (as a percentage of the payload)

The second term in the equation may be found using the Tsiolkovsky Rocket Equation (Turner, 2004).

\section{Conclusion}

The scope for Interplanetary Exploration is wider now than it has ever been in the history of our species. Unfortunately, this comes at a time when there is no other alternative than to do so in order to simply protect ourselves and what might be the only life forms in this entire universe. So, for the solution of this regrettable predicament, this paper provides an extremely cheap, efficient and effective method for the design and development of space launch vehicles, as this is probably our best bet on survival if the ever-shrinking resources are taken into account. It also gives a complete manual on how to use the equation, its specifications, how it should be applied and also how, with the help of it and a harmonious work relationship between human beings and machines, we can completely change the celestial stalemate that has gripped us since the moon landings in 1969. As a note of importance, this author believes the significance of this equation in the field of rocket design is immense: Since it combines all possible variables in a foolproof manner, it opens up the possibility of designing and developing rockets without the need for models and extensive preliminary, trial-and-error testing, hopefully reducing development costs by up to $95 \%$. Finally, it provides a detailed account of the processes by which the end result was reached, reinforcing the ideal that above all discovery lies the need for a voice of reason.

\section{Acknowledgement}

I acknowledge and thank Professor Muhammad M. H. Talukder, Ph.D. and Ms. Shabnam Munir, M.Phil. for their invaluable inspiration and encouragement.

\section{Author's Contributions}

This article was entirely prepared, developed and carried out by Zaheen Abdur Rahman.

\section{Ethics}

This article is original and contains unpublished material. The corresponding author confirms that there are no ethical issues involved.

\section{References}

Hitchens, F.E., 2015. The Encyclopedia of Aerodynamics. 1st Edn., Andrews UK Limited, Luton, ISBN-10: 1785383248, pp: 877.

Johnson and Cutnell, 1995. Physics: 3rd Edn., Wiley and Sons, New Jersey, USA. ISBN-10: 9780471898504 , pp: 47.

NASA, 1977. In: Chapter 6: Building the Colony and Making it Prosper; Estimating Costs and Time; Table 6.1: Personnel Requirements; $\mathrm{L}_{5}$ colony. Space Settlements A Design Study, NASA SP-413. National Aeronautics and Space Administration, USA. ISBN: 9781410218223. pp: 122.

NASA, 2002. Human Spaceflight. Space Shuttle Requirements. National Aeronautics and Space Administration.

Silver, J., 2009. 125 Physics Projects for the Evil Genius. 1st Edn., The McGraw-Hill Companies, Inc., New York, USA, ISBN-10: 9780071626071, pp: 29. 
Turner, M.J.L., 2004. Expedition Mars. 1st Edn., Springer Science and Business Media, London, UK, ISBN-10: 1852337354, pp: 321.

\section{Appendix}

${ }^{1}$ According to Newton's Second Law of Motion, Force $=$ Mass $*$ Acceleration, which, as shall be found later on, is $2 \mathrm{~g}$, or $19.6133 \mathrm{~m} \mathrm{~s}^{-2}$.

${ }^{2}$ The height at which the divider is placed is determined by the Relative Atomic Mass (R.A.M.) of the two fuels, using the equation $\frac{A_{r}}{M_{r}} *\left(\frac{4 \pi R^{3}}{3}+\frac{m-M}{D}\right)=H \pi R^{2}+\frac{2 \pi R^{3}}{3}$, with the same variables as in Equation 7 and Equation 8 and $H$ being the height of the cylinder from its bottom at which the divider is put, $A_{r}$ being the R.A.M. of the lower fuel and $M_{r}$ being the R.F.M. of the total fuel.

${ }^{3}$ This is found by the fuel quantity divided by the density and inner circle's area.

${ }^{4}$ This is found by the Equation $\frac{4 D \pi R^{3}}{3}=M$, with the same variables as in Equation 7.

${ }^{5}$ This is found by $\pi r^{2} h d$.

${ }^{6} 0.5 \mathrm{~m}$, since we want the average ' $\mathrm{m}$ ', that can only be $\frac{1 m+0 m}{2}$, which shall be $0.5 \mathrm{~m}$.

${ }^{7}$ Speed is distance by time, so it is $g n(n+1) \div n$. So, average speed must be $g(n+1)$, or $\left(g s_{r}+1\right)$.

${ }^{8}$ by two, since we want the average ' $\rho$ ', that can only be $\frac{(1.225+0)}{2}$, which shall be $0.6125 \mathrm{~kg} \mathrm{~m}^{-3}$, or $0.5 \mathrm{~atm}$.

${ }^{9} \mathrm{~m}$ is total fuel, so it must be $\mathrm{kg} \mathrm{s}^{-1}$ times the total time. 